\title{
ESTUDO PROSPECTIVO SOBRE SISTEMAS AUTOMÁTICOS DE DETECÇÃO, ALARME E SUPRESSÃO DE INCÊNDIOS
}

Geovana Pires Araujo Lima ${ }^{1}$, Josiane Dantas Viana Barbosa ${ }^{2}$, Juliano Zaffalon Gerber $^{3}$

1 Senai CIMATEC; Avenida Orlando Gomes, 1845, Piatã; Salvador/BA; gpalima@uesc.br

${ }^{2}$ Senai CIMATEC; Avenida Orlando Gomes, 1845, Piatã; Salvador/BA;

${ }^{3}$ Universidade Estadual de Santa Cruz; Rodovia Jorge Amado, km 16; Ilhéus/BA;

Resumo: Este artigo apresenta uma revisão bibliométrica e de conteúdo sobre os sistemas de monitoramento, detecção, alarme e extinção de incêndio. Para tanto selecionou-se a base Web of Science, e o gerenciamento das referências foi feito por meio do EndNote Web. A partir dos descritores e filtros usados, foi obtido um grupo amostral de 43 artigos. Estes artigos foram analisados, evidenciando que os estudos publicados, em sua maioria, apresentam uma abordagem experimental, devido à importância fulcral do aspecto funcional, o agente extintor mais citado foi a água atomizada e em aderência com as tendências da Industria 4.0, as pesquisas exibiram forte integração com tecnologias wireless, Internet das coisas, e robôs com inteligência artificial embarcada.

Palavras-Chave: Monitoramento; incêndio; extinção; proteção.

\section{PROSPECTIVE STUDY ABOUT AUTOMATIC FIRE DETECTION, ALARM, AND SUPPRESSION SYSTEMS}

Abstract: This article presents a bibliometric and content review of fire monitoring, detection, alarm and extinguishing systems. For this purpose, the Web of Science database was selected and the references were managed through EndNote Web. From the descriptors and filters used, a sample group of 43 articles was obtained. These papers were analyzed, showing that most published studies present an experimental approach, due to the central importance of the functional aspect, the most cited extinguishing agent was atomized water and in line with Industry 4.0 trends, research showed strong integration with wireless technologies, loT, and embedded artificial intelligence robots.

Keywords: Monitoring; fire; extinction; protection. 


\section{INTRODUÇÃO}

Incêndios e suas temíveis consequências, evidenciam a necessidade do desenvolvimento e implementação de efetivos sistemas de proteção. $[1,2,3,4,5,6,7,8]$ evidenciam essa afirmativa apresentando exemplos de desastres provocados pelo incêndio.

Em contrapartida, $[9,10,11,12]$ argumentam que a maioria das tragédias poderiam ter sido evitadas, ou pelo menos atenuadas, se houvessem sistemas efetivos de monitoramento, detecção, alarme e extinção de incêndio.

Neste tipo de sistema, o tempo é fator fulcral, com isso, é fundamental garantir a rápida detecção para que sejam acionados alarmes e os sinais de saída para que a extinção aconteça de forma imediata e segura. Outro ponto importante é garantir a assertividade da detecção, com o intuito de evitar falso-positivo.

Com isso, diversas linhas de pesquisas estão sendo desenvolvidas para contribuir de maneira efetiva em situações de incêndio. Desta forma, para mapear e entender o desenvolvimento científico nesta área, foi iniciada um estudo prospectivo por meio de uma combinação de técnicas de análise bibliométrica e de conteúdo.

\section{METODOLOGIA}

De acordo com [13] os tipos de pesquisas podem apresentar diferentes classificações quanto à finalidade, natureza, forma de abordagem, objetivos e procedimento técnicos. Desta forma, a Tabela 1 apresenta a classificação do presente estudo.

Tabela1 - Classificação do estudo

\begin{tabular}{|l|l|}
\hline \multicolumn{1}{|c|}{ CLASSIFICAÇÃO } & ENQUADRAMENTO DO ESTUDO \\
\hline Quanto à finalidade & Pesquisa aplicada ou tecnológica \\
\hline Quanto à natureza & Pesquisa observacional \\
\hline Quanto à forma de abordagem & Pesquisa qualitativa \\
\hline Quanto aos objetivos & Pesquisa exploratória \\
\hline Quanto aos procedimentos técnicos & Pesquisa bibliográfica \\
\hline
\end{tabular}

Fonte: Adaptado de [13]

O procedimento empregado para a realização do estudo é representado na Figura 1. Inicialmente foi definido o objetivo do trabalho, que consiste em mapear e entender o desenvolvimento científico dos sistemas de monitoramento, detecção, alarme e extinção de incêndio, a partir de um estudo bibliométrico e de conteúdo.

Para tal foi selecionado a base de dados Web of Science, pois possui ampla abrangência internacional e com cobertura multidisciplinar. $O$ gerenciador de referências empregado foi o EndNote Web. Para obtenção dos resultados utilizou-se operadores booleanos e filtros como limitação do período de busca, e configuração das áreas de busca. 
Figura 1- Fluxograma do método empregado

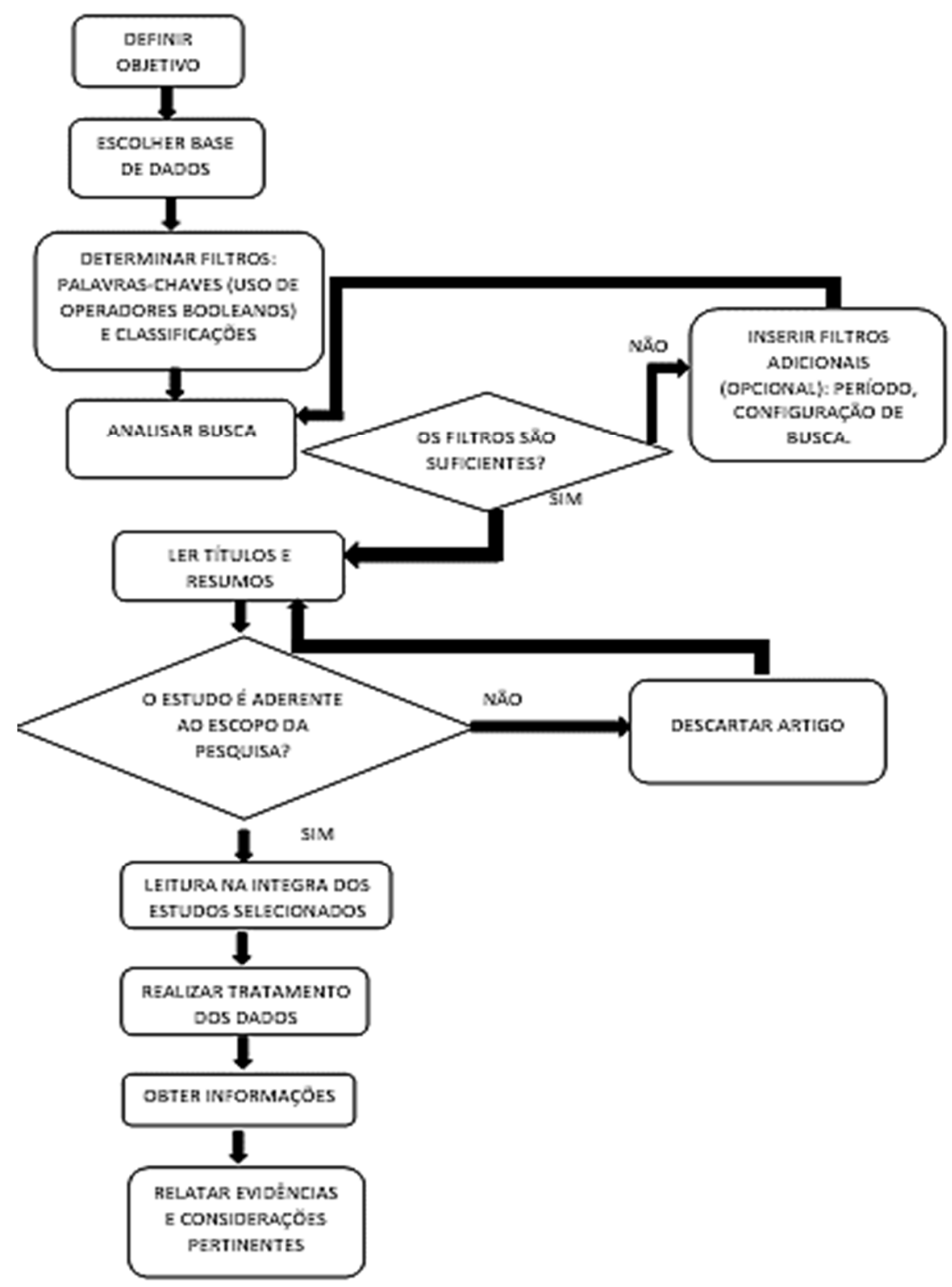

Fonte: autoria própria

Após a aplicação dos filtros, foi feita a leitura dos títulos e resumos, os estudos que não se enquadravam dentro do escopo da foram descartados. A partir do grupo amostral selecionado, foi realizado a análise bibliométrica por meio da avaliação estatística dos resultados, com o auxílio dos recursos do Web of Science. Os campos examinados foram: as principais áreas de pesquisa, número de publicações em função do ano, os principais países que publicam na área e a também foi relacionada a relações das palavras chaves. Que foi engendrada com o auxílio do software VOSwier, este permite a visualização em rede da co-ocorrência de termos importantes, ou palavras-chaves, que são extraídos da literatura científica observada. Além disso, foi feita a análise do conteúdo, a partir da leitura na integra do material selecionado. $\mathrm{O}$ que possibilitou compreender o desenvolvimento científico e as suas tendências. 


\section{\begin{tabular}{l|l} 
CIRCULAR ECONOMY & ECONOMIA CIRCULAR
\end{tabular}}

\section{RESULTADOS E DISCUSSÃO}

A partir do método supracitado, as análises foram feitas e os resultados obtidos. A Figura 2 ilustra o procedimento empregado para seleção do grupo amostral.

Figura 2- Resultado da busca na literatura

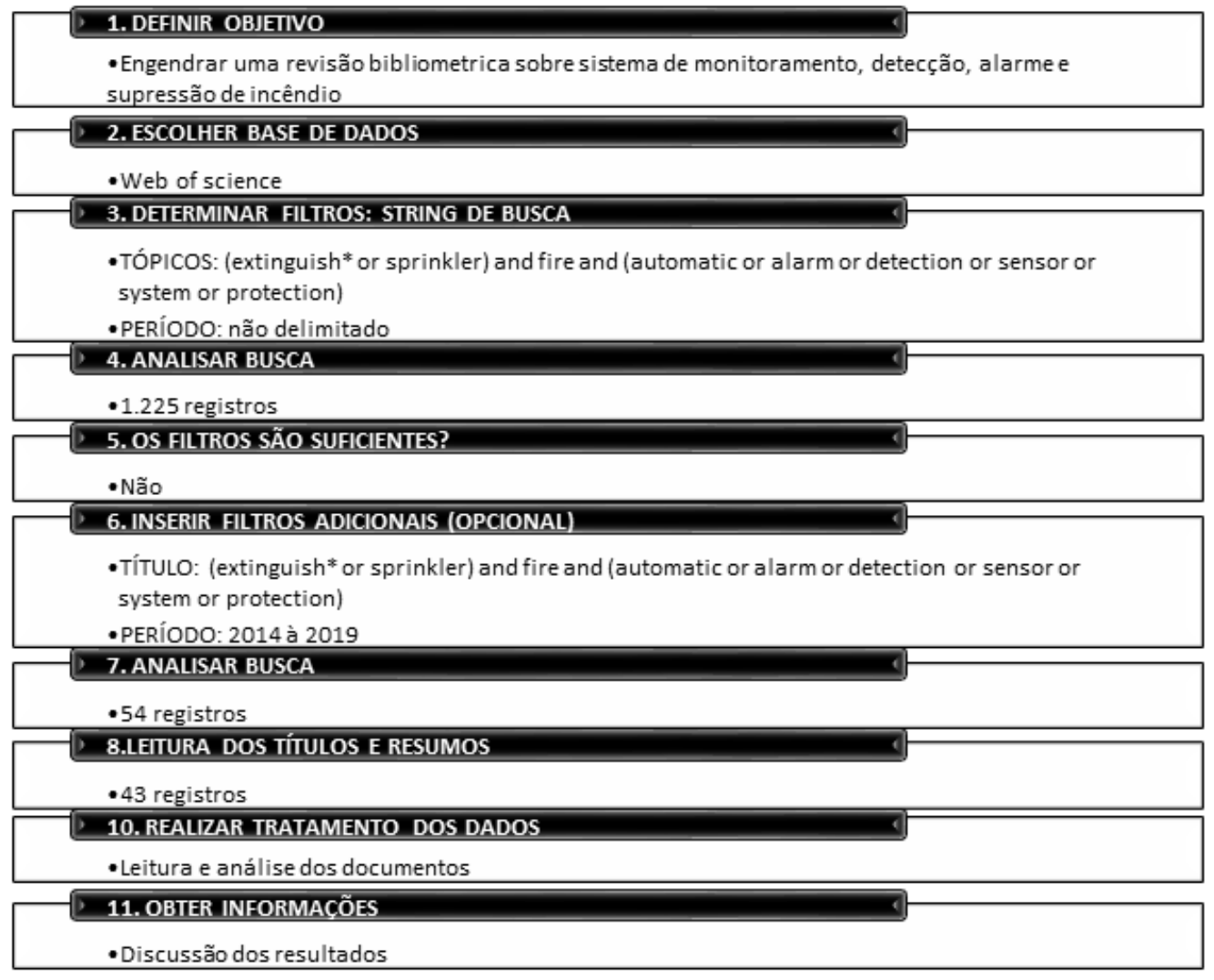

Fonte: autoria própria

O grupo amostral compreendido no presente escopo foi de 43 artigos. Inicialmente foi feita a análise bibliométrica, com foco no número de artigos publicados em função do ano, apresentado na Figura 3 (a), e as principais áreas de publicação são ilustradas na Figura 3 (b).

Figura 3- (a) Porcentagem de artigos por ano(b) Áreas de publicação
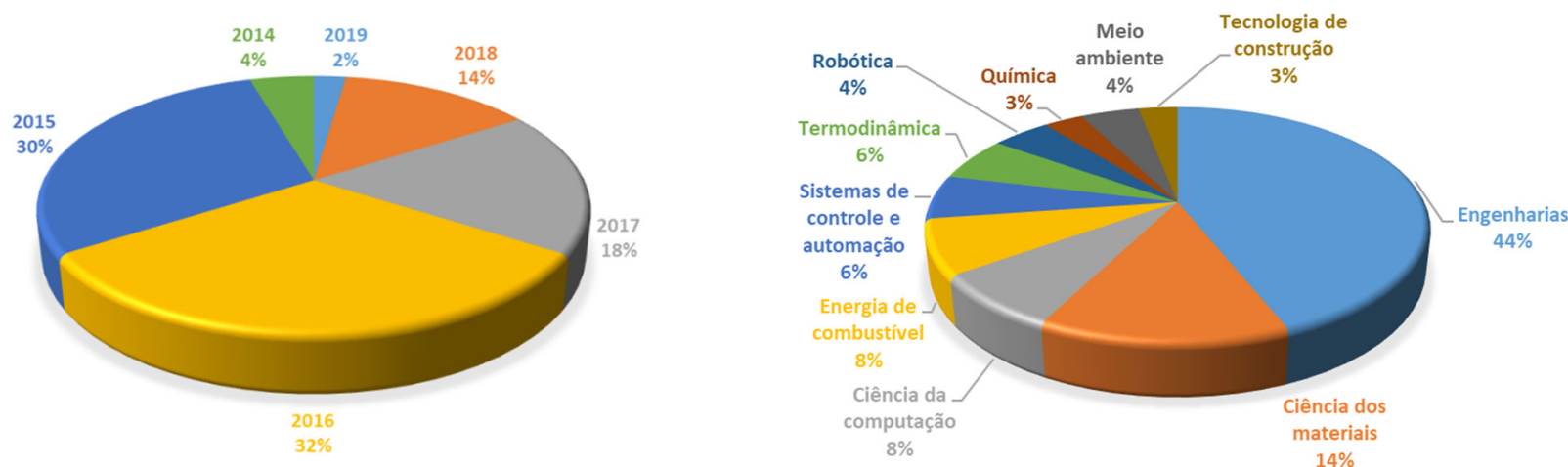

Fonte: autoria própria 
Observa-se na Figura 3 que o ano de 2016 apresenta maior densidade de publicações, para o grupo amostral analisado. É importante salientar que antes da aplicação dos filtros e seleção do material a partir da leitura dos títulos e resumos, a tendência do número de publicações em relação ao ano, apresenta comportamento crescente, evidenciando que a área é emergente. Verifica-se que o ano de 2019 apresenta um baixo número de publicações, isso é devido ao fato de que o período abrangido pelo estudo foi de abril a julho de 2019. Desta forma, não é possível inferir afirmações referente ao comportamento de 2019. A Figura 3 (b) apresenta as principais áreas de pesquisa relacionadas com a busca, sendo as Engenharias, o principal campo de pesquisa, o que é aderente com o esperado, pois o desenvolvimento dos sistemas estudados perpassa por diversas áreas da Engenharia, e aplicação do desenvolvimento tecnológico.

Além disso, foram identificados os países que mais publicam na área, tendo a China com maior número de pesquisas publicadas, seguido da Rússia, Estados Unidos, Coreia do Sul e Índia.

Figura 4- Países que mais publicam na área examinada

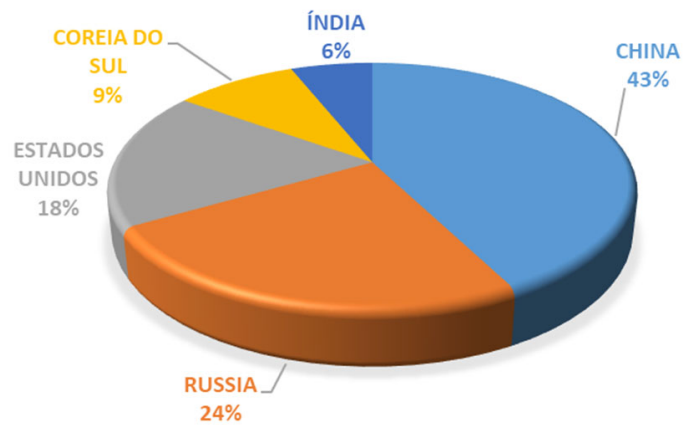

Fonte: autoria própria.

Para examinar a relação entre os termos mais importantes, foi construída uma rede com o auxílio do software VOSwier. Ilustrada na Figura 5.

Figura 5- Rede de correlação entre os principais termos das pesquisas analisadas

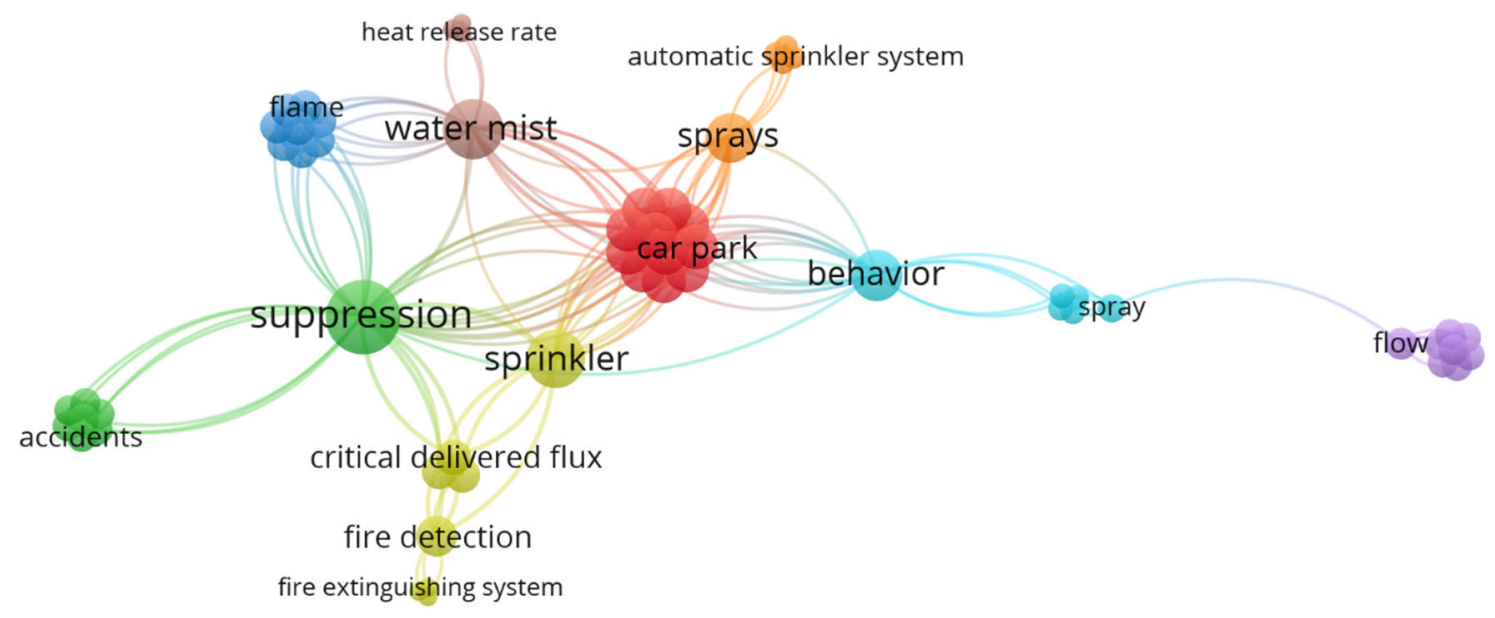

Fonte: autoria própria. 
A rede de palavras-chaves apresentada na Figura 5 mostra as correlações, a partir de laços, entre os termos mais importantes utilizado no grupo amostral estudado. Os principais termos da rede são: supressão, sprinkler, detecção de fogo, água atomizada e fogo.

A avaliação do conteúdo, realizada a partir da leitura de cada pesquisa do grupo amostral, permitiu observar que a principal abordagem utilizada nos estudos foi a experimental, isso se deve, possivelmente, pelo fato de que a funcionalidade deste tipo de sistema é um aspecto fulcral. O agente extintor que foi mais citado foi a água atomizada, em que segundo [14], este sistema possui uma série de vantagens, que justificam a sua utilização, como por exemplo, não é tóxico, nem para pessoas, nem para o ambiente; mais seguro para as pessoas, se comparado com agente de supressão a base de $\mathrm{CO}_{2}$; pode ser compactado; ocupa pouco espaço; apresenta bom custo benefício, entre outros.

Além disso, verificou-se o desenvolvimento robótico, para a substituição do homem, devido ao alto risco que a atividade promove. Então, com o intuito de evitar a exposição do ser humano em situações de alto rico, estão sendo desenvolvidos robôs com inteligência artificial embarcada, para poderem atuar de maneira eficiente na extinção de incêndios. Convergindo com as tendências da Indústria 4.0.

Seguindo esta tendência, também foi observado que diversas pesquisas se baseiam no uso de tecnologia wireless, sem fio, para a conexão dos sistemas de monitoramento, detecção, alarme e extinção de incêndios. No entanto, a norma ABNT NBR 17.240, que reporta sobre os sistemas de detecção e alarme, não contempla o uso de sistemas wireless. Não sendo convergente, portanto, com o desenvolvimento científico e a com os conceitos da Indústria 4.0. O risco de uma falha de detecção na presença de incêndio apresenta elevada criticidade, no entanto, as tecnologias sem fio estão cada vez mais eficazes, e pode-se usar métodos redundantes para garantir a eficiência na detecção e evitar falso-positivo.

\section{CONCLUSÃO}

Diante do exposto, pode-se concluir que os objetivos propostos foram atendidos. A partir do escopo da pesquisa, verifica-se que a área analisada se configura como tecnologia emergente, devido ao aumento de publicação ao longo dos anos. Entre os 43 artigos selecionados, observou-se que a maior densidade de publicação ocorreu no ano de 2016 , com 14 publicações. Os países que estão à frente do desenvolvimento científico destes sistemas são: China, Rússia, Estados Unidos, Coreia do Sul e Índia, respectivamente. E o principal campo do conhecimento envolvido diz respeito às áreas das Engenharias, presente em 29 dos 43 artigos analisados.

Por meio da análise de conteúdo do grupo amostral examinado, de 43 artigos, observou-se que a abordagem metodológica mais utilizada pelos pesquisadores foi a experimental, pois a funcionalidade é o aspecto mais crítico deste tipo de sistema. $O$ agente supressor mais citado foi a água atomizada, devido suas vantagens em comparação com outros agentes. E em conformidade com a indústria 4.0, muitas 
pesquisas apresentam o uso de tecnologias como wireless, internet das coisas- loT, Inteligência artificial- Al, robotização, entre outras, para a melhoria ou desenvolvimento de novos produtos. No entanto, no que tange os aspectos normativos brasileiros, verifica-se que a norma que versa sobre sistemas de detecção e alarme, não contempla o uso de tecnologias sem fio, apresentando divergência com a tendência de desenvolvimento tecnológico.

Para trabalhos futuros, sugere-se estender a revisão de literatura para outras bases além da Web of Science.

\section{Agradecimentos}

Fapesb pelo apoio financeiro BOL n 3014/2019.

\section{REFERÊNCIAS}

${ }^{1}$ MENDONÇA, H. T. T. Edificações civis em situação de incêndio: estudo de caso da boate Kiss e do edifício Joelma. Trabalho de Conclusão de Curso. Engenharia Civil. Centro Universitário de Formiga - UNIFOR-MG, 2014.

${ }^{2}$ HAHNEMANN, A. L. C.; CORREAA, C.; RABBANI E. R. K. Fire safety evaluation: an alternative method for Brazilian buildings. Revista ALCONPAT. Asociación Latinoamericana de Control de Calidad, Patología y Recuperación de la Construcción, A. C. e-ISSN: 2007-6835. DOI: http://dx.doi.org/10.21041/ra.v7i2.178. Revista ALCONPAT. 2007,

${ }^{3}$ ANTUNES, M. A. G. ; GOUVEIA, A. M. C. . A gestão de riscos como alternativa de prevenção de incêndio em edificação arquivística: estudo de caso. Revista Internacional de Desastres Naturales, Accidentes e Infraestructura Civil , 2012.

${ }^{4} \mathrm{ONO}, \quad \mathrm{R}$. Proteção do Patrimônio histórico-cultural contra incêndio em edificações de interesse de preservação. Palestra apresentada na Fundação Casa de Rui Barbosa. Rio de Janeiro, 2004.

${ }^{5}$ SÁ, D. M. D., SÁ, M. R., \& LIMA, N. T. O Museu Nacional e seu papel na história das ciências e da saúde no Brasil. Cadernos de Saúde Pública, v. 34, p. e00192818, 2018.

${ }^{6}$ VANNUCCI, P., MASI, F., STEFANOU, I., \& MAFFI-BERTHIER, V. Structural integrity of Notre Dame Cathedral after the fire of April 15 ${ }^{\text {th }}$. Archive ouverte HAL, 2019.

${ }^{7}$ STANKUNAVICIUS, A. Brasil precisa de maior proteção contra incêndios. Revista Incêndio, 2015. Disponível em: < http://revistaincendio.com.br/brasil-precisa-demaior-protecao-contra-incendios/ > Acesso em: 15 de agosto de 2018. 
${ }^{8}$ RAMOS, M. Prejuízo da Ultracargo com incêndio em Santos é de R\$ 1 bilhão. Revista Época, $2015 . \quad$ Disponível em: $<$ https://epoca.globo.com/tempo/expresso/noticia/2015/04/prejuizo-da-ultracargocom-incendio-em-santos-e-de-r-1-bilhao.html> Acesso em: 15 de agosto de 2018.

${ }^{9}$ MARIO, L. Análise comparativa de custos para as diferentes ocupações de risco no sistema aberto de chuveiros automáticos. Trabalho de conclusão de especialização, Universidade Federal do Rio Grande do Sul. Escola de Engenharia. Curso de Engenharia de Segurança do Trabalho, 2013.

${ }^{10}$ ALMEIDA, A. K.; FRANZOLOSO, C. R. G. Projetos de Proteção e Combate a Incêndio: Segurança Como Requisito Fundamental. Revista UNICIÊNCIAS, v. 19, n. $1,2015$.

${ }^{11}$ BUTRY, D. T.; BROWN, M. H.; FULLER, S.K. Benefit-cost analysis of residential fire sprinkler systems. US Department of Commerce, National Institute of Standards and Technology, 2007.

${ }^{12} \mathrm{KIM}$, J.; LEE, S. Forecasting and identifying multi-technology convergence based on patent data: the case of IT and BT industries in 2020. Scientometrics, v. 111, n. 1, p. 47-65, 2017.

${ }^{13}$ FONTELLES, M. J., SIMÕES, M. G., FARIAS, S. H., \& FONTELLES, R. G. S. . Metodologia da pesquisa científica: diretrizes para a elaboração de um protocolo de pesquisa. Revista Paraense de Medicina, v. 23, n. 3, p. 1-8, 2009.

${ }^{14}$ MACEDO, W. M., OLIVEIRA, H. L. S. Uso do sistema de water mist para ações de combate a incêndios em unidades marítimas de produção. Congresso nacional de Excelência em Gestão.X CONGRESSO NACIONAL DE EXCELÊNCIA EM GESTÃO 08 e 09 de agosto de 2014. Rio de Janeiro- RJ. 